\title{
The theory of exciton states in quasi-zero-dimensional semiconductor systems
}

\author{
S.I. Pokutnyi \\ Illichivsk Institute of the Mechnikov Odessa National University, \\ 17A, Danchenko str., Illichivsk, Odessa Reg., 68001 Ukraine \\ E-mail: Pokutnyi_Sergey@inbox.ru \\ Phone: +380(4868) 4-30-76; fax: +380(4868) 6-01-54
}

\begin{abstract}
For a semiconductor quantum dot (QD), the contributions made to the exciton energy spectrum by the electron and hole kinetic energies, the energy of Coulomb interaction between them, and the energy of their polarization interaction with the spherical interface between the QD and the dielectric medium have been analyzed.
\end{abstract}

Keywords: exciton state, semiconductor quantum dot, exciton energy spectrum.

Manuscript received 10.04.06; accepted for publication 23.10.06.

\section{Introduction}

Optical properties of quasi-zero-dimensional semiconductor structures, consisting of the semiconductor QDs of the spherical shape with radii $a \approx 1 \ldots 10 \mathrm{~nm}$, grown in transparent dielectric media have been intensively studied recently [1-5]. Such heterostructures attract attention owing to their nonlinear optical properties and the prospects of their application in optoelectronics and quantum electronics, in particular, as novel materials perspective for the creation of elements which control optical signals in semiconductor injection lasers $[1,2]$ and in optical bistable elements and transistors [2].

Since the energy gap in a semiconductor QD is essentially narrower than that in semiconductor (dielectric) matrices, the motion of charge carriers is confined to the QD volume in all three directions, i.e., charge carriers move in a three-dimensional spherical potential well. As a result, both an electron and a hole as well as an exciton have no quasi-momenta in a QD. Therefore, it is possible to speak only about quasiparticle states in a QD. Below, as regards to excitons in a QD, we understand such an exciton state that has no quasi-momentum.

Optical and electrooptical properties of similar heterophase systems are determined to a great extent by the energy spectrum of a spatially-bounded electron-hole pair (the exciton) [1-5]. The energy spectrum of the charge carriers in a QD will be completely discrete for the QD dimension $a$ smaller than that of the order of the Bohr radii of an electron $a_{e}$ and a hole $a_{h}$ [6-8]. Under these conditions, the influence of the interface between the QD and the dielectric matrix can cause the dimensional quantization of the electron and hole energy spectra in the QD, which is related to both the mere spatial confinement of a quantization region $[4,5,9]$ and the polarization interaction of charge carriers with the QD surface [3, 6-14].

The theory of exciton states in quasi-zerodimensional structures has not yet been sufficiently developed so far. To fill this gap, the contributions to the exciton energy spectrum, made by the electron and hole kinetic energies and the Coulomb interaction energies between them, as well as the energy of their polarization interaction with the spherical interface between the QD and the dielectric medium, have been analyzed in this paper. In addition, the limit transition from the energy spectrum of the exciton in the QD to that of the exciton in the unlimited bulk has been traced.

The exciton, the structure (the effective mass, Bohr radius, and bond energy) of which in the QD does not differ from that in an infinite semiconductor, will be called as the "bulk" exciton.

\section{Exciton energy spectrum in a quasi-zero- dimensional system}

Following papers [3, 6-14], let us consider a simple model of the quasi-zero-dimensional system: a neutral spherical semiconductor QD of the radius $a$ and dielectric permittivity (DP) $\varepsilon_{2}$ imbedded into a dielectric matrix with DP $\varepsilon_{1}$. In the bulk of such a QD, 
moving are an electron $e$ and a hole $h$ with effective masses $m_{e}$ and $m_{h}$, respectively. The variables $r_{e}$ and $r_{h}$ denote the distances of the electron and the hole, respectively, from the center of the QD. The electron and hole bands are supposed parabolic. The typical dimensions of the problem are the quantities $a, a_{e}, a_{h}$, and $a_{\text {ex }}$, where

$$
\begin{aligned}
& a_{e}=\left(\varepsilon_{2} \hbar^{2} / m_{e} e^{2}\right), a_{h}=\left(\varepsilon_{2} \hbar^{2} / m_{h} e^{2}\right), \\
& a_{\mathrm{ex}}=\left(\varepsilon_{2} \hbar^{2} / \mu e^{2}\right)
\end{aligned}
$$

are the Bohr radii of the electron, hole, and exciton, respectively, in an infinite semiconductor with DP $\varepsilon_{2} ; e$ is the electron charge; and $\mu=\left(m_{e} m_{h} /\left(m_{e}+m_{h}\right)\right)$ is the reduced effective mass of the exciton. The circumstance that all the typical dimensions of the problem are considerably larger than the interatomic distance $a_{0}$ :

$$
a, a_{e}, a_{h}, a_{\mathrm{ex}}>>a_{0},
$$

allows us to consider the motions of the electron and the hole in the QD in the effective mass approximation.

In the model concerned, the Hamiltonian of the exciton in the QD, in the framework of the approximations stated above, looks like [10-14]

$$
\begin{aligned}
& H=-\frac{\hbar^{2}}{2 m_{e}} \Delta_{e}-\frac{\hbar^{2}}{2 m_{h}} \Delta_{h}+ \\
& +E_{g}+V_{e h}\left(r_{e}, r_{h}\right)+U\left(r_{e}, r_{h}, a\right),
\end{aligned}
$$

where the first two terms define the kinetic energies of the electron and the hole, and $E_{g}$ is the energy gap width in an unconfmed semiconductor with DP $\varepsilon_{2}$. In Eq. (3), the energy of the electron-hole Coulomb interaction $V_{e h}\left(r_{e}, r_{h}\right)$ is defined as

$$
V_{e h}\left(r_{e}, r_{h}\right)=-\frac{e^{2}}{\varepsilon_{2}\left|r_{h}-r_{e}\right|}
$$

Provided that $\varepsilon_{1}>>\varepsilon_{2}$, the polarization interaction energy $U\left(r_{e}, r_{h}, a\right)$ in Eq. (3) can be written down as an algebraic sum of the energies of the hole and electron interactions with their own images, $V_{h h^{\prime}}\left(r_{h}, a\right)$ and $V_{e e^{\prime}}\left(r_{e}, a\right)$, respectively, and with the images of "foreign" quasi-particle $V_{e h^{\prime}}\left(\vec{r}_{e}, \vec{r}_{h}, a\right)=V_{h e^{\prime}}\left(\vec{r}_{e}, \vec{r}_{h}, a\right)$ [10-14]:

$$
\begin{aligned}
& U\left(\vec{r}_{e}, \vec{r}_{h}, a\right)=V_{h h^{\prime}}\left(r_{h}, a\right)+V_{e e^{\prime}}\left(r_{e}, a\right)+ \\
& +V_{e h^{\prime}}\left(\vec{r}_{e}, \vec{r}_{h}, a\right)+V_{h e^{\prime}}\left(\vec{r}_{e}, \vec{r}_{h}, a\right),
\end{aligned}
$$

where

$$
V_{h h^{\prime}}\left(r_{h}, a\right)=\frac{e^{2}}{2 \varepsilon_{2} a}\left(\frac{a^{2}}{a^{2}-r_{h}^{2}}+\frac{\varepsilon_{2}}{\varepsilon_{1}}\right),
$$

$$
\begin{aligned}
& V_{e e^{\prime}}\left(r_{e}, a\right)=\frac{e^{2}}{2 \varepsilon_{2} a}\left(\frac{a^{2}}{a^{2}-r_{e}^{2}}+\frac{\varepsilon_{2}}{\varepsilon_{1}}\right), \\
& V_{e h^{\prime}}=V_{h e^{\prime}}=-\frac{e^{2}}{2 \varepsilon_{2} a} \cdot \frac{a}{\left[\left(r_{e} r_{h} / a\right)^{2}-2 r_{e} r_{h} \cos \Theta+a^{2}\right]^{1 / 2}},
\end{aligned}
$$

$\Theta=\angle \vec{r}_{e}, \vec{r}_{h}$.

Although, in our model of the quasi-zerodimensional system, the electron and the hole do not go beyond the space of the semiconductor QD, the potential energy of their interaction with the spherical interface of two media $U\left(r_{e}, r_{h}, a\right)$ (5) depends not only on the DP $\varepsilon_{2}$ of the QD, but also on the DP $\varepsilon_{1}$ of the matrix, into which the QD is imbedded [3, 10-14]. Such a dependence is connected to the penetration of the electrostatic field created by the electron and the hole beyond the boundaries of the QD.

In our papers [10-14], when considering Hamiltonian (3) of the exciton in the QD, the polarization interaction (5) of charge carriers with the surface charge induced by them at the spherical interface "QD-dielectric matrix" was taken into account for the first time. Later on, such a polarization interaction was taken into account when calculating the exciton $[3,10]$ and biexciton [9] energy spectra in the QD.

On the basis of papers [10-14], we will obtain the energy spectrum of an exciton in a QD making use of the approximation, where the QD is an infinitely deep spherical potential well for an electron and a hole that move inside its space. The radius $a$ of the QD is taken as confined within the limits

$a_{h}<<a \leq a_{e} \approx a_{\mathrm{ex}}$.

Then, the polarization interaction (5) plays a dominating role in the potential energy of Hamiltonian (3). Provided that condition (9) holds true, we use the adiabatic approximation, supposing the kinetic energy of an electron to have the largest value and considering the last two terms in Hamiltonian (3), as well as the operator of nonadiabaticity, in the framework of the perturbation theory.

Confining ourselves to the first order of the perturbation theory, we obtain the following expression for the exciton energy spectrum $E_{n_{e}, l_{e}=0, m_{e}=0}^{n_{h}, l_{h}, m_{h}=0}(S)$ in the state $\left(n_{e}, l_{e}=0, m_{e}=0 ; n_{h}, l_{h}, m_{h}=0\right)$ in the QD with the radius $S[3,10-14]$ :

$E_{n_{e}, 0,0}^{n_{h}, l_{h}, 0}(S)=E_{g}+T_{n_{e}, l_{e}=0}^{e}(S)+\bar{V}_{e e^{\prime}}(S)+\lambda_{n_{e}, 0,0}^{n_{h}, l_{h}, 0}(S)$,

where

$T_{n_{e}, 0}^{e}(S)=E_{n_{e}, 0}^{e}(S)=\frac{\pi^{2} n_{e}^{2}}{S^{2}}$

is the kinetic energy of the electron in the infinitely deep spherical well, $\bar{V}_{e e^{\prime}}(S)$ is the average value of the interaction energy of the electron with its own image 
calculated using the wave functions of the electron in the infinitely deep spherical well of the QD,

$$
\lambda_{n_{e}, 0,0}^{t_{h}}(S)=\frac{P_{n_{e}, 0}}{S}+\omega\left(S, n_{e}\right)\left(t_{h}+\frac{3}{2}\right)
$$

is the oscillator-type hole spectrum,

$$
\begin{aligned}
& \frac{P_{n_{e}, 0}}{S}=\bar{V}_{h h^{\prime}}(S)+\left(\bar{V}_{e h}^{n_{e}, 0,0}(S)\right)^{\prime}+ \\
& +\left(\bar{V}_{e h^{\prime}}^{n_{e}, 0,0}(S)+\bar{V}_{h e^{\prime}}^{n_{e}, 0,0}(S)\right),
\end{aligned}
$$

$\omega\left(S, n_{e}\right)=2\left(1+\frac{2}{3} \pi^{2} n_{e}^{2}\right)^{1 / 2}\left(\frac{m_{e}}{m_{h}}\right)^{1 / 2} S^{-3 / 2}$

is the frequency of oscillations of the hole, $t_{h}=2 n_{r_{h}}+l_{h}=0,1,2, \ldots$ is the main quantum number of the hole, and $n_{r_{h}}=0,1,2, \ldots$ is the radial quantum numbers of the hole. Provided that the hole energy spectrum $\lambda_{n_{e}, 0,0}^{t_{h}}(S)$ (12) can be described by the spectrum of a three-dimensional harmonic oscillator, the requirement

$$
S^{1 / 2} \gg\left(\frac{m_{e}}{m_{h}}\right)^{1 / 2} \frac{t_{h}+3 / 2}{\left(1+\frac{2}{3} \pi^{2} n_{e}^{2}\right)^{1 / 2}}
$$

must be fulfilled [10-14].

Let us write down the expressions for the average values of the energy of the electron interaction with its own image [10-14]

$$
\begin{aligned}
& \bar{V}_{e e^{\prime}}^{n_{e}, 0,0}(S)=\frac{Z_{n_{e}, 0}}{S}, \\
& \left(Z_{n_{e}, 0}=\frac{\varepsilon_{2}}{\varepsilon_{1}}+2 \int_{0}^{1} \frac{d x \sin ^{2}\left(\pi n_{e} x\right)}{1-x^{2}}\right),
\end{aligned}
$$

the energy of the hole interaction with its own image

$\bar{V}_{h h^{\prime}}(S)=\frac{1+\left(\varepsilon_{2} / \varepsilon_{1}\right)}{S}$,

the energies of the electron and hole interactions with the images of "foreign" quasi-particle

$$
\bar{V}_{e h^{\prime}}^{n_{e}, 0,0}(S)+\bar{V}_{h e^{\prime}}^{n_{e}, 0,0}(S)=-\frac{2}{S},
$$

and the electron-hole Coulomb interaction energy

$$
\begin{aligned}
& \left(\bar{V}_{e h}^{n_{e}, 0,0}(S)\right)^{\prime}=-\frac{2}{S}\left[\ln \left(2 \pi n_{e}\right)+\gamma-\operatorname{Ci}\left(2 \pi n_{e}\right)\right], \\
& \widetilde{\bar{V}}_{e h}^{n_{e}, 0,0 ; t_{h}}(S)=\left(\bar{V}_{e h}^{n_{e}, 0,0}(S)\right)^{\prime}+\omega\left(S, n_{e}\right)\left(t_{h}+\frac{3}{2}\right)= \\
& =-\frac{2}{S}\left[\ln \left(2 \pi n_{e}\right)+\gamma-\operatorname{Ci}\left(2 \pi n_{e}\right)\right]+\omega\left(S, n_{e}\right)\left(t_{h}+\frac{3}{2}\right),
\end{aligned}
$$

where $\operatorname{Ci}(\gamma)$ is the cosine-integral function, $\gamma=0.577 \ldots$ is the Euler constant.

It should be noted that the formulae (12)-(19) were obtained by averaging the corresponding expressions (4), (6)-(8) using the wave functions of the infinitely deep spherical well of a QD [3, 10-14].

The polarization interaction energy (5), averaged using the electron wave functions in the infinitely deep spherical well, looks like

$\bar{U}_{\mathrm{pol}}^{n_{e}, 0,0}(S)=\bar{V}_{h h^{\prime}}(S)+\bar{V}_{e e^{\prime}}^{n_{e}, 0,0}(S)+$

$+\left(\bar{V}_{e h^{\prime}}^{n_{e}, 0,0}(S)+\bar{V}_{h e^{\prime}}^{n_{e}, 0,0}(S)\right)=$

$=\frac{Z_{n_{e}, 0}+\left(\varepsilon_{2} / \varepsilon_{1}\right)-1}{S}$.

Hereafter, the energy is measured in terms of $\mathrm{Ry}_{e}=\left(\hbar^{2} / 2 m_{e} a_{e}^{2}\right)$, and the dimensionless variables $x=\left(r_{h} / a\right)$ and $S=\left(a / a_{e}\right)$ are used.

Taking into account Eqs (12), (13) and (19), (20), we write down the expression (10) for the exciton spectrum $E_{n_{e}, 0,0}^{t_{h}}(S)$ in the state $\left(n_{e}, 0,0 ; t_{h}\right)$ in the QDs, the radii $S$ of which satisfy conditions (9) and (15) simultaneously, as follows:

$E_{n_{e}, 0,0}^{t_{h}}(S)=E_{g}+T_{n_{e}, 0}^{e}(S) \times$

$\times\left[1+\frac{\bar{U}_{\mathrm{pol}}^{n_{e}, 0,0}(S)}{T_{n_{e}, 0}^{e}(S)}-\frac{\left|\widetilde{\bar{V}}_{e h}^{n_{e}, 0,0} ; t_{h}(S)\right|}{T_{n_{e}, 0}^{e}(S)}\right]$.

It should be pointed out that the exciton spectrum (21) was obtained in the framework of the adiabatic approximation, where the kinetic energy of the electron $T_{n_{e}, 0}^{e}(S)$ (11) was supposed to give the main contribution to the energy spectrum of the exciton in the QD. Therefore, formula (21) for the exciton energy spectrum $E_{n_{e}, 0,0}^{t_{h}}(S)$ allows one to trace the contributions, given to the exciton spectrum by the electron-hole Coulomb interaction (19) and the polarization interaction (20) and to compare them with the contribution of the electron kinetic energy (11).

The obtained exciton spectrum (21) can be applied only to weakly excited exciton states $\left(n_{e}, 0,0 ; t_{h}\right)$, for which the inequality

$E_{n_{e}, 0,0}^{t_{h}}(S)-E_{g}<<\Delta V(S)$

where $\Delta V(S)$ is the depth of the potential well for electrons in the QD holds true (for example, in a CdS QD with dimensions obeying condition (9), the value of $\Delta V$ is $2.3 \ldots 2.5 \mathrm{eV}[15])$. 
In [14], the spectrum of an exciton in a QD of the radius $a$ for a simple model of the quasi-zerodimensional system, where the Hamiltonian of the exciton $H$ is given by the formula (3), was found using the variational method and not being restricted to the framework of the adiabatic approximation. Moreover, the radius $a$ of the QD, contrary to [3, 10-14], was not bounded by condition (9). The results of variational calculations of the energy spectrum for the exciton $E_{0}(a)$ in the QD of the radius $a$ are shown in the figure. The relevant calculation parameters in the work [14] corresponded to experimental conditions in the works $[4,5,15,16]$.

\section{Contributions of kinetic, polarization, and Coulomb energies to the spectra of excitons in quantum dots}

In papers $[4,16]$, the peaks of interband absorption in spherical QDs with the radius $a$ within the interval of $1.2 \ldots 30 \mathrm{~nm}$, which were made of CdS with DP $\varepsilon_{2}=9.3$ and dispersed in a transparent matrix of silicate glass with DP $\varepsilon_{1}=2.25$ [4], were observed. The effective masses of the electron and hole and the reduced mass of the exciton $\mu$ in CdS were $\left(m_{e} / m_{0}\right)=0.205$, $\left(m_{h} / m_{0}\right)=5$, and $\left(\mu / m_{0}\right)=0.197$, respectively [16]. In particular, the dependence of the positions of the adsorption band of QDs caused by interband transitions onto the dimensional quantization levels $\left(n_{e}=1, l_{e}=0\right),\left(n_{e}=1, l_{e}=1\right)$, and $\left(n_{e}=1, l_{e}=2\right)$ of the electron in the conduction band on the QD radius $a$ was experimentally determined $[4,16]$.

As was shown in $[3,10-14]$, the formula (21) describes the exciton spectrum $E_{1,0,0}^{t_{h}}(S)$ (5) as a function of the QD radius $S$ with sufficient accuracy under the reported conditions of experiments in the $\mathrm{CdS}$ $[4,16]$. The parameters of the exciton spectrum $E_{1,0,0}^{t_{h}}(S)$ (21) under the experimental conditions [4, 16] for the CdS QDs with the radius $a=1.5 \ldots 3.0 \mathrm{~nm}$ are listed in Table 1.

According to the formulae (11), (19), and (20), the ratios between the polarization interaction energy and electron kinetic energy $\left(\bar{U}_{\text {pol }}^{1,0,0}(S) / T_{1,0}^{e}(S)\right)$ as well as between the Coulomb interaction energy and electron kinetic energy $\left(\widetilde{\bar{V}}_{e h}^{1,0,0}(S) / T_{1,0}^{e}(S)\right)$ are proportional to $S$ and $S^{1 / 2}$, respectively. Such a behavior of the ratios $\left(\bar{U}_{\text {pol }}(S) / T_{1,0}^{e}(S)\right)(20)$ and $\left(\widetilde{\bar{V}}^{1,0,0 ; t_{h}}(S) / T_{1,0}^{e}(S)\right)$ (19) is also confirmed by numerical data in Table 1.

From Table 1, it follows that the polarization interaction energy $\bar{U}_{\text {pol }}^{1,0,0}(S)$ (20) makes the dominating contribution to the exciton energy spectrum (21), whereas the Coulomb interaction energy $\widetilde{V}_{e h}^{1,0,0 ; t_{h}}(S)$ (19) makes a small negative one. Namely, the ratio $\left(\bar{U}_{\text {pol }}^{1,0,0}(S) / T_{1,0}^{e}(S)\right)$ varies from $55.8 \%$ at $a=1.5 \mathrm{~nm}$ to $112 \%$ at $a=3 \mathrm{~nm}$, whereas the absolute value of the ratio $\left(\widetilde{V}_{e h}^{1,0,0 ; t_{h}}(S) / T_{1,0}^{e}(S)\right)$ from $8.5 \%$ at $a=1.5 \mathrm{~nm}$ to $30 \%$ at $a=3 \mathrm{~nm}$. The data presented in Table 1 are also confirmed by the results of variational calculations of the spectrum $E_{0}(a)$ of the exciton in the QD of the radius $a \leq 3 a_{\text {ex }}$, which were obtained in the work [14] under the experimental conditions of the works $[4,16]$ and beyond the adiabatic approximation.

The main contributions to the polarization interaction energy $\bar{U}_{\text {pol }}^{1,0,0}(S)$ (20) are made by the interaction energies of the electron $\bar{V}_{e e^{\prime}}^{1,0,0}(S)$ (16) $(64.5 \%)$ and the hole $\bar{V}_{h h^{\prime}}^{1,0,0}(S)(17)(58.2 \%)$ with their own images, whereas the interaction energy of the electron and hole with the images of "others" $\left(\bar{V}_{e h^{\prime}}^{1,0,0}(S)+\bar{V}_{h e^{\prime}}^{1,0,0}(S)\right) \quad$ (18) gives a negative contribution, the absolute value of which is $22.7 \%$ (see Table 2). It is essential that those contributions do not depend on the QD radius $S$.

Table 1. Contributions to the exciton spectrum $E_{1,0,0}^{t_{h}}(a)$ (10) and (21) made by the electron-hole Coulomb, $\left|\tilde{V}_{e h}^{1,0,0 ; t_{h}}(a)\right| \quad(19)$, and polarization, $\bar{U}_{\mathrm{pol}}^{1,0,0}(a)$ (20), interaction energies in relation to the contribution of the electron kinetic energy $T_{1,0}^{e}(a)(11)$.

\begin{tabular}{|c|c|c|c|c|c|}
\hline $\begin{array}{c}a(\mathrm{~nm}) \\
(S)\end{array}$ & $\begin{array}{l}T_{1,0}^{e}(S) \\
\left(\mathrm{Ry}_{e}\right)\end{array}$ & $t_{h}$ & 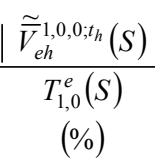 & $\frac{\bar{U}_{\mathrm{pol}}^{1,0,0}(S)}{T_{1,0}^{e}(S)}$ & $\begin{array}{l}{\left[E_{1,0,0}^{t_{h}}(S)-\right.} \\
\left.-E_{g}\right]\left(\mathrm{Ry}_{e}\right)\end{array}$ \\
\hline \multirow{2}{*}{$\begin{array}{c}1.5 \\
(0.624)\end{array}$} & \multirow{2}{*}{25.35} & 0 & 17.4 & 55.8 & 35.08 \\
\hline & & 1 & 8.5 & & 37.34 \\
\hline \multirow{2}{*}{$\begin{array}{c}2.0 \\
(0.83)\end{array}$} & \multirow{2}{*}{14.26} & 0 & 25.6 & $74 . .4$ & 21.21 \\
\hline & & 1 & 15.3 & & 22.68 \\
\hline \multirow{2}{*}{$\begin{array}{c}2.5 \\
(1.04)\end{array}$} & \multirow{2}{*}{9.13} & 0 & 34.0 & 93.0 & 14.51 \\
\hline & & 1 & 22.5 & & 15.56 \\
\hline \multirow{2}{*}{$\begin{array}{c}3.0 \\
(1.25)\end{array}$} & \multirow{2}{*}{6.34} & 0 & 42.6 & 111.5 & 10.71 \\
\hline & & 1 & 30.0 & & 11.51 \\
\hline
\end{tabular}

Note. The ratio $\widetilde{\bar{V}}_{e h}^{1,0,0 ; t_{h}}(a) / T_{1,0}^{e}(a)$ is negative. The data are listed for the CdS QDs with the radii $a=(1.5 \ldots 3.0) \mathrm{nm}$ under the conditions of experiments in $[4,16]$. $\mathrm{Ry}_{e}=7.68 \cdot 10^{-1} \mathrm{eV}$. 
Table 2. Contributions to the polarization interaction energy $\bar{U}_{\mathrm{pol}}^{1,0,0}(a)(20)$ made by the interaction of the electron with its own image, $\bar{V}_{e e^{\prime}}^{1,0,0}(a)(16)$, by the interaction of the hole with its own image, $\bar{V}_{h h^{\prime}}^{1,0,0}(a)(17)$, and by the interactions of the electron and the hole with the hole and electron images, respectively, $\left|\bar{V}_{e h^{\prime}}^{1,0,0}(a)+\bar{V}_{h e^{\prime}}^{1,0,0}(a)\right|(18)$, as well as the ratio $\left|\tilde{\bar{V}}_{e h}^{1,0,0 ; t_{h}}(a) / \bar{U}_{\text {pol }}^{1,0,0}(a)\right|$ between the Coulomb and polarization interaction energies (19) and (20).

\begin{tabular}{|c|c|c|c|c|c|c|}
\hline $\begin{array}{c}a(\mathrm{~nm}) \\
(S)\end{array}$ & $\begin{array}{c}\bar{U}_{\text {pol }}^{1,0,0}(S) \\
\left(\mathrm{Ry}_{e}\right)\end{array}$ & $\begin{array}{c}\frac{\bar{V}_{e e^{\prime}}^{1,0,0}(S)}{\bar{U}_{\mathrm{pol}}^{1,0,0}(S)} \\
(\%)\end{array}$ & $\begin{array}{c}\frac{\bar{V}_{h h^{\prime}}^{1,0,0}(S)}{\bar{U}_{\text {pol }}^{1,0,0}(S)} \\
(\%)\end{array}$ & $\frac{\bar{V}_{e h^{\prime}}^{1,0,0}(S)+\bar{V}_{h h^{\prime}}^{1,0,0}(S) \mid}{U_{\mathrm{pol}}^{1,0,0}(S)}\left(\frac{1 \%)}{(\%)}\right.$ & $t_{h}$ & $\begin{array}{c}\widetilde{\bar{V}}_{e h}^{1,0,0 ; t_{h}}(S) \\
\bar{U}_{\text {pol }}^{1,0,0}(S) \\
(\%)\end{array}$ \\
\hline \multirow{2}{*}{$\begin{array}{c}1.5 \\
(0.624)\end{array}$} & \multirow{2}{*}{14.14} & \multirow{2}{*}{64.5} & \multirow{2}{*}{58.2} & \multirow{2}{*}{22.7} & 0 & 31.2 \\
\hline & & & & & 1 & 15.2 \\
\hline \multirow{2}{*}{$\begin{array}{c}2.0 \\
(0.83) \\
\end{array}$} & \multirow{2}{*}{10.61} & \multirow{2}{*}{64.5} & \multirow{2}{*}{58.2} & \multirow{2}{*}{22.7} & 0 & 34.4 \\
\hline & & & & & 1 & 20.5 \\
\hline \multirow{2}{*}{$\begin{array}{c}2.5 \\
(1.04)\end{array}$} & \multirow{2}{*}{8.49} & \multirow{2}{*}{64.5} & \multirow{2}{*}{58.2} & \multirow{2}{*}{22.7} & 0 & 36.6 \\
\hline & & & & & 1 & 24.2 \\
\hline \multirow{2}{*}{$\begin{array}{c}3.0 \\
(1.25) \\
\end{array}$} & \multirow{2}{*}{7.07} & \multirow{2}{*}{64.5} & \multirow{2}{*}{58.2} & \multirow{2}{*}{22.7} & 0 & 38.2 \\
\hline & & & & & 1 & 26.9 \\
\hline
\end{tabular}

Note. The ratios $\left(\left(\bar{V}_{e h^{\prime}}^{1,0,0}(S)+\bar{V}_{h e^{\prime}}^{1,0,0}(S)\right) / U_{\text {pol }}^{1,0,0}(S)\right)$ and $\left(\widetilde{\bar{V}}_{e h}^{1,0,0 ; t_{h}}(S) / \bar{U}_{\text {pol }}^{1,0,0}(S)\right)$ are negative. The data are listed for the CdS QDs with radii $a=1.5 \ldots 3.0 \mathrm{~nm}$ under the conditions of experiments in $[4,16]$.

The Coulomb interaction energy $\widetilde{\bar{V}}_{e h}^{1,0,0 ; t_{h}}(S)$ (19) makes a considerably smaller contribution to the excilon spectrum (10) and (21) in comparison with the polarisation interaction energy $\bar{U}_{\text {pol }}^{1,0,0}(S)(20)$. The ratio of these energies $\left(\widetilde{V}_{e h}^{1,0,0 ; t_{h}}(S) / \bar{U}_{\text {pol }}^{1,0,0}(S)\right)$ becomes negative (its absolute value changes from 31 and $15 \%$ at $a=1.5 \mathrm{~nm}$ to 38 and $27 \%$ at $a=3 \mathrm{~nm}$ for $t_{h}=0$ and 1 , respectively (see Table 2 ).

The experimental exciton spectrum was stated in papers $[4,16]$ to be described with sufficient accuracy by the kinetic energy of the electron in the QD $T_{1,0}^{e}(a)(11)$ as the radius $a$ of the CdS QD increases above $2.0 \mathrm{~nm}$. Actually, as follows from Table 1, the ratio $\left(\left|\bar{U}_{\text {pol }}^{1,0,0}(S)+\widetilde{\bar{V}}_{e h}^{1,0,0 ; t_{h}}(S)\right| / T_{1,0}^{e}(S)\right)$ of the sum of the polarization and Coulomb interaction energies to the kinetic energy of the electron comprises a significant value of (0.49-0.69). Even for the QD with the smallest experimentally allowable radius $a=1.5 \mathrm{~nm}$, such a ratio amounts to a substantial value of about $38 \%$ (see the figure).

\section{Emergence of the bulk exciton in a quasi-zero-dimensional system}

For the QD with a small radius $a<<a_{\mathrm{ex}}$, the main contribution to the exciton spectrum (21) is given by the electron kinetic energy $T_{n_{e}, l_{e}}^{e}(S)$ (11), whereas the contributions of the terms $\bar{U}_{\mathrm{pol}}^{n_{e}, 0,0}(S)$ (5) and $\widetilde{\bar{V}}_{e h}^{n_{e}, 0,0 ; t_{h}}(S)$ are small [7-14]:

$\bar{U}_{\mathrm{pol}}^{\mathrm{n}_{\mathrm{e}}, 0,0}(S) / T_{n_{e}, l_{e}}^{e}(S)<<1$,

$\left|\widetilde{\bar{V}}_{e h}^{n_{e}, 0,0 ; t_{h}}(S) / T_{n_{e}, l_{e}}^{e}(S)\right|<<1$.

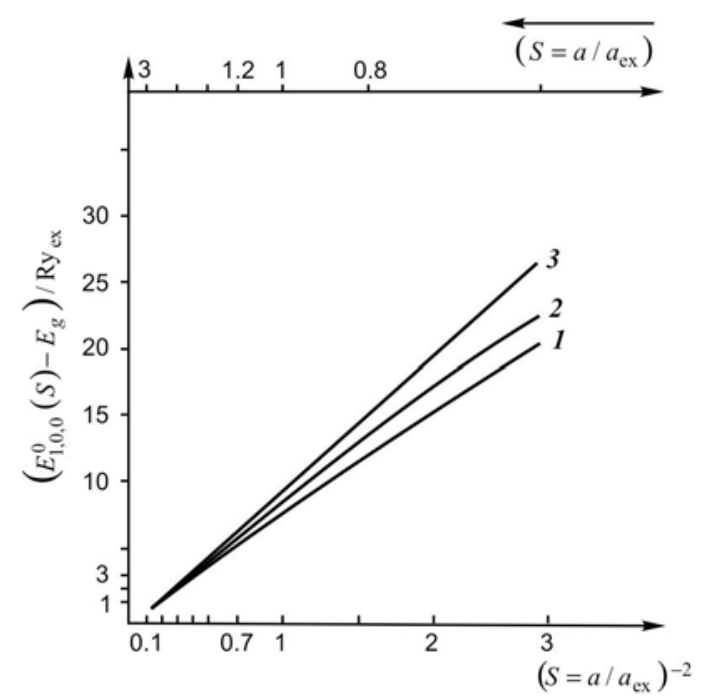

The exciton energy spectra $E_{1,0,0}^{0}(S)$ as functions of the nanocrystal dimension $S=a / a_{\mathrm{ex}}: 1$ - the experimental exciton spectrum $[4,16], 2$ - the exciton spectrum $E_{0}(a)$ obtained by the variational method [14], 3 - the kinetic energy of the electron $T_{1,0}^{e}(S)(11)$. 
For the QD with a large radius $a>>a_{\mathrm{ex}}$, the exciton is quantized as a whole, and its energy spectrum is determined as [9]

$$
\begin{aligned}
& E_{n l}(a)=E_{g}-E_{\mathrm{ex}}+\frac{\hbar^{2}}{2 M a^{2}} \varphi_{n l}^{2}, \\
& E_{\mathrm{ex}}=-\mathrm{Ry}_{\mathrm{ex}}=-\frac{\hbar^{2}}{2 \mu a_{\mathrm{ex}}^{2}},
\end{aligned}
$$

where $M$ is the translational mass of the exciton, and $\varphi_{n l}$ are the roots of the Bessel function $J_{l+\frac{1}{2}}(\varphi)$.

In paper [17], an expression for the exciton spectrum was obtained by the variational method in the framework of the effective mass approximation but without regard for the polarization interaction energies of an electron and a hole with the surface of the QD, which made it possible to trace the limit transition to the spectrum of the bulk exciton (22), starting from the QD radius $S_{\mathrm{ex}}>S_{\mathrm{ex}}^{0} \geq 8$.

The spectrum of the exciton in the CdS QD, which was found by us in the paper [14] by the variational method in the framework of the effective mass approximation by taking into account the polarization interaction energy, turns into the spectrum of the bulk exciton (22) at $S_{\text {ex }}>\widetilde{S}_{\text {ex }}^{0} \geq 9.44$ under the experimental conditions of the works $[4,5,16]$. In this case, the value of $\widetilde{S}_{\mathrm{ex}}^{0}$ differs from that of $S_{\mathrm{ex}}^{0}$ by no more than $18 \%$. Such a difference is connected to the fact that the account of the polarization interaction energy (it has not been done in the paper [4]) results in the exciton energy growth proportional to $S^{-1}$. In addition, the values of the QD radii $S_{\mathrm{ex}}^{0}$ and $\widetilde{S}_{\mathrm{ex}}^{0}$, can be overestimated to a certain extent, because the variational calculations of the exciton spectrum yield the overestimated values of the energy.

\section{Conclusions}

Here, in the framework of the simple model of a quasizero-dimensional system, we have shown that, even for the QDs with the smallest experimentally allowable radii, the kinetic energy of the electron (11) makes a contribution to the exciton spectrum $E_{n_{e}, 0,0}^{t_{h}}(a)$ (10) and (21) that is comparable by the order of magnitude with the contributions made to this spectrum by the polarization, $\bar{U}_{\mathrm{pol}}^{\mathrm{n}_{\mathrm{e}}, 0,0}(a) \quad(20), \quad$ and Coulomb, $\widetilde{\bar{V}}_{e h}^{n_{e}, 0,0, t_{h}}(a)$ (19), interaction energies. In this connection, a description of the exciton spectrum in QDs with radii $a<a_{\mathrm{ex}}$ using only the expression for the electron kinetic energy $T_{n l}^{e}(a)(11)$, as it has been done in works $[4,5,16]$, is not justified.

In the least studied case where the QD radius $a$ is comparable by its value with the Bohr radius of the exciton, we showed [10-14] that the exciton spectrum $E_{n_{e}, 0,0}^{t_{h}}(a)$ (10) and (21) can be described by the complicated dependence

$$
E_{n_{e}, 0,0}^{t_{h}}(a)=f\left(a^{-1}, a^{-3 / 2}, a^{-2}, m_{e}^{-1}, m_{h}^{-1}\right)
$$

(see the figure). In this case, the effective masses of the electron $m_{e}$ and the exciton $\mu$ are the functions of the QD radius $a$ [12-14].

\section{References}

1. J.I. Alferov // Fiz. Tekhn. Poluprovodn. 32, p. 3 (1998) (in Russian) (Semiconductors 32, p. 3 (1998)).

2. N.N. Ledentsov, V.M. Ustinov, V.A. Shchukin et al. // Fiz. Tekhn. Poluprovodn. 32, p. 385 (1998) (in Russian) (Semiconductors, 32, p. 263 (1998)).

3. S.I. Pokutnyi // Theory of excitons in quasi-zerodimensional semiconducting systems. Astroprint, Odesa, 2003 (in Russian).

4. A.I. Ekimov, A.A. Onushchenko // Pis'ma Zh. Eksp. Teor. Fiz. 40, p. 337 (1984) (in Russian) (JETP Lett. 40, p. 1136 (1984)).

5. A.I. Ekimov, A.A. Onushchenko, Al.L. Efros // Pis'ma Zh. Eksp. Teor. Fiz. 43, p. 292 (1986) (in Russian) (JETP Lett. 43, p. 376 (1986)).

6. N.A. Efremov, S.I. Pokutnyi // Fiz. Tverd. Tela 27, p. 48 (1985) (in Russian) (Sov. Phys. Solid State 27, p. 27 (1985)).

7. N.A. Efremov, S.I. Pokutnyi // Fiz. Tverd. Tela 32, p. 2921 (1990) (in Russian) (Sov. Phys. Solid State 32, p. 1697 (1990)).

8. S.I. Pokutuyi // Fiz. Tekhn. Poluprovodn. 31, p. 1443 (1997) (in Russian) (Semiconductors 31, p. 1247 (1997)).

9. Al.L. Efros, A.L. Efros // Fiz. Tekhn. Poluprovodn. 16, p. 1209 (1982) (in Russian) (Semiconductors 16, p. $955(1982))$.

10. N.A. Efremov, S.I. Pokutnyi // Fiz. Tverd. Tela 32, p. 1637 (1990) (in Russian) (Sov. Phys. Solid State 32, p. 955 (1990)).

11. S.I. Pokutnyi // Fiz. Tekhn. Poluprovodn. 25, p. 628 (1991) (in Russian) (Semiconductors 25, p. 381 (1991)).

12. S.I. Pokutnyi // Phys. Lett. A. 203, p. 388 (1995).

13. S.I. Pokutnyi // Fiz. Tekhn. Poluprovodn. 30, p. 1952 (1996) (in Russian) (Semiconductors 30, p. 1015 (1996)).

14. S.I. Pokutnyi // Fiz. Tverd. Tela, 38, p. 2667 (1996) (in Russian) (Phys. Solid State 38, p. 1463 (1996)).

15. V.Ya. Grabovskis, Ya.Ya. Dzenis, A.L. Ekimov // Fiz. Tverd. Tela 31, p. 272 (1989) (in Russian) (Sov. Phys. Solid State 31, p. 1255 (1989)).

16. D. Chepik, A. Efros, A. Ekimov // J. Lumin. 47, p. 113 (1990).

17. N.V. Tkach, V.A. Golovatskii // Preprint. Institute of Theoretical Physics, Acad. of Sci. of Ukr.SSR, 90-59, Kiev, 1990 (in Russian). 